\title{
La formación inicial de profesores en Chile: 'Voces' de la comunidad chilena de investigación en educación matemática
}

\author{
The initial education of teachers in Chile: 'voices' of the Chilean community of \\ research in Mathematics Education \\ A formação inicial de professores no Chile: 'Vozes' da comunidade chilena de \\ pesquisa em educação matemática
}

Luis R. Pino-Fan

luis.pino@ulagos.cl

Universidad de Los Lagos

Osorno, Chile

\author{
Ismenia Guzmán-Retamal \\ ismenia.guzman@ulagos.cl \\ Universidad de Los Lagos \\ Santiago, Chile \\ Macarena Larraín \\ mlarrainj@uandes.cl \\ Universidad de los Andes \\ Santiago, Chile \\ Claudia Vargas-Díaz \\ mlarrainj@uandes.cl \\ Universidad de Santiago de Chile \\ Santiago, Chile
}

Recibido-Received: 12/ago/2016 / Corregido-Corrected: 1/dic/2016.

Aceptado-Accepted: 21/mar/2017 / Publicado-Published: 31/ene/2018.

\begin{abstract}
Resumen
En este artículo presentamos los puntos de vista de 17 personas investigadoras chilenas reconocidas, a nivel nacional e internacional, por sus contribuciones al campo de la educación matemática, y concretamente a la formación de profesorado de matemáticas. Para ello, se elaboró una encuesta con la cual exploramos las opiniones y reflexiones de personal experto sobre las características de los actuales programas de formación de profesorado de matemáticas en Chile. Como resultado de este estudio, se plantean algunas recomendaciones que podrían tenerse en cuenta para el mejoramiento de los programas de formación docente, que permitan, así, oportunidades para la adquisición de competencias didáctico-matemáticas idóneas para la gestión de los aprendizajes.
\end{abstract}

Palabras claves: Formación de profesorado; plan de estudios; competencias docentes; personal experto. 
ISSN Electrónico: 2215-3470

DOI: http://dx.doi.org/10.15359/ru.32-1.5
UNICIENCIA Vol. 32, No. 1, pp. 68-88. Enero-Junio, 2018. URL: www.revistas.una.ac.cr/uniciencia Email: revistauniciencia@una.cr

\begin{abstract}
In this article, we present the viewpoints of 17 nationally and internationally recognized Chilean researchers for their contributions to the field of mathematics education, and specifically to the training of mathematics teachers. For such purpose, a survey that explored the opinions and reflections of experts about the characteristics of the current mathematics teachers training programs in Chile was conducted. As a result of this study, some recommendations were proposed; they could be considered for the improvement of teacher training programs, allowing opportunities for acquisition of suitable didactic-mathematical competences for the management of the learning.
\end{abstract}

Keywords: Teacher education; Curriculum; Teacher qualifications; Experts.

\title{
Resumo
}

Neste artigo apresentamos os pontos de vista de 17 pessoas pesquisadoras chilenas reconhecidas em nível nacional e internacional por suas contribuições no campo da educação matemática e, concretamente na formação do professorado de matemática. Para isso, elaborou-se uma enquete com a qual exploramos as opiniões e reflexões de pessoas peritas sobre as características dos atuais programas de formação de professorado de matemática no Chile. Como resultado deste estudo, planteiam-se algumas recomendações que poderiam ser levadas em consideração para a melhoria dos programas de formação docente, que permitam, assim, oportunidades para a aquisição de competências didático-matemática idôneas para a gestão das aprendizagens.

Palavras-chaves: Formação de professorado; plano de estudos; competências docentes; pessoas peritas.

El estudio sobre los conocimientos que debería tener un profesor o profesora de matemáticas para desempeñarse de manera eficiente en el aula es un tema de investigación que no es nuevo, pero que en años recientes ha ido ganando cada vez más atención tanto de la comunidad internacional de investigación en didáctica de las matemáticas, como de las administraciones educativas y organismos abocados a la política educativa a nivel mundial (véase por ejemplo, Ball, Thames \& Phelps, 2008; Kaiser, Bloemeke, Busse, Doehrmann \& Koenig, 2014; Pino-Fan \& Godino, 2015; Shulman, 1986). La principal razón del creciente interés es que el desarrollo del pensamiento y de las competencias matemáticas del alumnado depende, fundamentalmente, de la enseñanza recibida $y$, por ende, de la formación de su respectivo profesorado.

Diversos estudios muestran un panorama de lo que ha sido la historia de la formación de docentes en Chile, cuáles han sido los cambios, los programas de mejoramiento educativo y las reformas (e.g., Ávalos, 2001; Cisternas, 2007; Cisternas, 2011; Contreras, 2016; Contreras-Sanzana \& Villalobos-Clavería, 2010; Latorre, 2006). En dichos estudios se refleja que los procesos de cambio para el mejoramiento de la formación inicial de docentes estuvieron motivados por problemas tales como la desarticulación entre las actividades de formación teórica y práctica en los programas de formación, la falta de espacios para la reflexión -por parte del futuro profesorado- de la actividad docente, la desarticulación entre los contenidos disciplinares y pedagógicos, por mencionar algunos.

Por su parte, la UNESCO (2012), a partir de un estudio realizado sobre docentes en América Latina y el Caribe, identificó cinco nudos críticos para mejorar los procesos de formación inicial docente. Dichos nudos refieren a la necesidad de: 1) seleccionar o atraer a mejores postulantes a las carreras de pedagogía; 2) elevar la calidad de los programas formativos y vincularlos adecuadamente con las demandas del mundo escolar; 3) preparación del personal formador de docentes; 4) formar docentes con preparación para atender a estudiantes de sectores más vulnerables y de minorías étnicas; y 5) establecer mecanismos de regulación y control de la calidad de los programas, de las instituciones formadoras y de sus egresados y egresadas. 
UNICIENCIA Vol. 32, No. 1, pp. 68-88. Enero-Junio, 2018.

ISSN Electrónico: 2215-3470

URL: www.revistas.una.ac.cr/uniciencia

Email: revistauniciencia@una.cr

En concordancia con las orientaciones políticas propuestas por la UNESCO (2012), y con el objetivo de fortalecer la calidad de los programas de formación de profesorado, el Ministerio de Educación de Chile, a través del Centro de Perfeccionamiento, Experimentación e Investigaciones Pedagógicas (CPEIP), impulsó la elaboración de un conjunto de estándares orientadores para carreras de pedagogía en educación (parvularia, especial, básica y media). Los estándares fueron publicados en el año 2012, y se organizaron en torno a dos grandes categorías: estándares pedagógicos y estándares disciplinarios. De acuerdo con el Ministerio de Educación (2012), los estándares pedagógicos corresponden a áreas de competencias genéricas de la función docente, las cuales son necesarias para la enseñanza de las disciplinas. En relación con los estándares disciplinarios, en el caso concreto de matemáticas y tomando como ejemplo los estándares de enseñanza media, se presentan organizados en torno a cinco áreas temáticas: Sistemas numéricos y álgebra; cálculo; estructuras algebraicas; geometría; y datos y azar. Para cada una de estas áreas temáticas se proponen estándares que el profesorado de enseñanza media debería cumplir al término de su formación inicial, los cuales están relacionados con el desarrollo y dominio de competencias matemáticas más que con competencias didáctico-matemáticas necesarias para la gestión de los aprendizajes del alumnado sobre dicha disciplina. De esta manera, pareciera que los estándares orientadores para la formación de profesorado, propuestos por el Ministerio de Educación de Chile en el 2012, establecen una separación latente entre lo disciplinario (las matemáticas en nuestro caso) y lo pedagógico, dando pie a que tanto el futuro profesorado como el recién egresado no realicen conexiones entre las distintas dimensiones del conocimiento didáctico-matemático requerido para la gestión idónea de los aprendizajes de sus estudiantes (ver, por ejemplo, las propuestas de Hill, Ball \& Schilling, 2008; Pino-Fan, Assis \& Castro, 2015; Rowland, Huckstep \& Thwaites, 2005; Schoenfeld \& Kilpatrick, 2008; Tatto et al., 2008).

De manera paralela, y también en sintonía con lo propuesto por la UNESCO (2012) y las tendencias internacionales, el Ministerio de Educación de Chile, a través del CPEIP, estableció un examen nacional: la Prueba Inicia. Esta prueba pretende evaluar contenidos disciplinares y pedagógicos, tomando como base los estándares propuestos por el mismo Ministerio. La prueba es aplicada desde el año 2012 a egresados de las carreras de pedagogía con el objetivo de "asegurar la calidad" de la formación inicial del profesorado (Cádiz \& Cordella, 2015; 'Prueba Inicia', 2012). Sin embargo, diversos estudios han evidenciado los resultados poco favorables respecto del objetivo de dicha prueba. Por ejemplo, Contreras (2016) señala:

En la misma línea, la prueba INICIA muestra que los futuros profesores de ciencias, se encuentran muy por debajo de los estándares esperados. En las carreras de pedagogía en ciencias (matemática, biología, física y química) sólo el $7 \%$ de los futuros profesores que rindieron la prueba de conocimientos disciplinares alcanza un nivel sobresaliente (2012). Luego, si observamos las especialidades en ciencias experimentales estos niveles de insuficiencia aumentan a un promedio de $74 \%$ en conocimiento disciplinar, donde el 55\% tiene además un nivel solo aceptable en los conocimientos pedagógicos. La tendencia en estos resultados se puede atribuir a diversos aspectos, entre ellos el evidente bajo conocimiento disciplinar de profesores y futuros profesores, pero un aspecto sobre el cual ha versado la discusión e investigación, es el referido al pensamiento sobre cómo enseñar los contenidos de las disciplinas de especialización. (p. 16) 
ISSN Electrónico: 2215-3470

DOI: http://dx.doi.org/10.15359/ru.32-1.5
UNICIENCIA Vol. 32, No. 1, pp. 68-88. Enero-Junio, 2018.

URL: www.revistas.una.ac.cr/uniciencia Email: revistauniciencia@una.cr

Si bien estos esfuerzos constituyen un gran avance y son innovadores en la región, considerando que los estándares orientadores y la Prueba Inicia forman parte de un programa de mejoramiento de la calidad de la formación inicial docente, su puesta en marcha requiere atender y supervisar el desarrollo de cada fase del proceso. La evaluación de la implementación pretende identificar potenciales diferencias entre lo planificado y la manera en que realmente está funcionando el programa, y debe considerar la evaluación de todos sus elementos (Tejedor, 2000). En este sentido, las visiones del personal formador de profesorado acerca de cómo se han estado implementando los estándares y la Prueba Inicia, resultan de gran interés para informar la evaluación general del programa. Así, es necesario evaluar y monitorear la implementación, que siempre es compleja y presenta nuevos desafíos que deben atenderse y abordarse, para que la política sea exitosa.

En particular, resulta interesante indagar sobre la manera y medida en que los planes de estudio de los programas de formación de profesorado en Chile se han ido adaptando a las sugerencias y recomendaciones de los estándares orientadores propuestos por el Ministerio en 2012. Así mismo, resulta relevante conocer los problemas que enfrentan los actuales programas y cómo estos pueden ser superados. Del mismo modo, cabe preguntarse si la Prueba Inicia evalúa efectivamente los conocimientos y competencias didáctico-matemáticas del futuro profesorado, necesarios para la gestión idónea de los aprendizajes de sus futuros y futuras estudiantes.

En este sentido, el objetivo de este trabajo es conocer las visiones de quienes investigan y forman docentes de matemáticas en Chile, y reflexionar en torno a dichas problemáticas, con la finalidad de plantear recomendaciones para el mejoramiento de los actuales programas de formación de profesorado de matemáticas en el país. Al mismo tiempo esto podría servir como experiencia para otros países de la región, que se encuentran en procesos de implementación de políticas de este tipo.

Para lograr nuestro objetivo, realizamos una encuesta a 17 personalidades investigadoras chilenas reconocidas a nivel nacional e internacional por sus contribuciones al campo de la educación matemática, y concretamente a la formación de profesorado de matemáticas. Esta investigación versa sobre el análisis de las respuestas y reflexiones que, a propósito de la encuesta, proporcionaron. De esta forma, con el análisis de las respuestas y reflexiones del personal experto, se responde a la pregunta ¿cuál es la situación actual y hacia dónde debemos avanzar en materia de la formación inicial de docentes de matemáticas en Chile?

\section{Metodología}

A partir de registros de las universidades chilenas y bases de datos nacionales tales como la de la Sociedad Chilena de Educación Matemática, fue posible identificar un total de 29 sujetos investigadores con perfil deseable para este estudio -i.e., pertenecer al ámbito de investigación sobre educación matemática o didáctica de la matemática, haber desarrollado investigación sobre la formación de profesorado, estar colaborando de manera activa en alguno de los programas nacionales sobre formación de profesorado de matemáticas e, idealmente, que pertenezcan al claustro de docentes de programas de postgrado en nuestra disciplina-. Se extendió una invitación a las 29 personas, vía correo electrónico o llamadas telefónicas, explicándoles la intencionalidad y los alcances que se pretendían con este estudio.

De las 29 personas investigadoras invitadas, 12 declinaron la invitación, aceptando participar en el estudio 17. De las 17 que aceptaron, al momento de llevar a cabo la encuesta, solo uno de ellos tenía el grado de Magíster en Didáctica de la Matemática y estaba próximo a obtener el 
UNICIENCIA Vol. 32, No. 1, pp. 68-88. Enero-Junio, 2018.

Email: revistauniciencia@una.cr

grado de Doctor en la misma especialidad. El resto de participantes poseía el grado de Doctor con especialidad en Didáctica de la Matemática, Educación Matemática o similar. La Tabla 1 presenta las universidades de adscripción de los 17 sujetos participantes.

Tabla 1

Universidades de adscripción de participantes

\begin{tabular}{lc}
\hline \multicolumn{1}{c}{ Universidad } & Investigadores/as \\
\hline Pontificia Universidad Católica de Chile & 2 \\
Pontificia Universidad Católica de Valparaíso & 2 \\
Universidad Católica de la Santísima Concepción & 1 \\
Universidad Católica del Maule & 1 \\
Universidad Católica del Norte & 2 \\
Universidad de Concepción & 1 \\
Universidad de los Andes & 1 \\
Universidad de Los Lagos & 2 \\
Universidad de Magallanes & 1 \\
Universidad de Santiago de Chile & 2 \\
Universidad del Bío-Bío & 1 \\
Universidad Metropolitana de Ciencias de la Educación & 1 \\
\hline & $n=17$ \\
\hline
\end{tabular}

Nota: Elaboración propia.

Para la recolección de la información, se elaboró una encuesta (Cohen, Manion \& Morrison, 2011) con la tecnología que proporciona Google Forms, la cual fue enviada por medio de correo electrónico a las 17 personas participantes. Una de las ventajas de usar la herramienta 'Google Forms' es que permite responder la encuesta de forma virtual y las respuestas son registradas automáticamente en una base de datos controlada por el equipo autor de este artículo. Así mismo, permite que los sujetos participantes se tomen el tiempo que consideren pertinente para reflexionar y responder las preguntas.

El diseño de las preguntas incorporadas en la encuesta constó de tres etapas. Primero, tomando en cuenta las sugerencias de bibliografía sobre el tema (e.g., lo discutido en la primera sección de este artículo), el equipo de investigación escribió la primera versión de las preguntas, que tomó en cuenta lo propuesto por Tejedor (2000) en relación con la evaluación de programas. A continuación, para garantizar la validez de la encuesta, esta fue sometida a un estudio mediante juicio de personal experto, en el que participaron cinco investigadores con amplia trayectoria en el campo de investigación sobre formación de profesorado de matemáticas adscritos a universidades de los siguientes países: México (1), Colombia (2), y España (2). Sobre la base de dicho estudio, se analizaron -y corrigieron- aspectos tales como la claridad y redacción de las 
cuestiones, y si con dichas cuestiones efectivamente se indagaban los aspectos centrales para los fines y propósitos de nuestra investigación. De forma paralela al estudio, mediante el juicio de personal experto, se realizó un "pilotaje" de las preguntas, con tres casos (cuyas respuestas no fueron contempladas para el análisis final que aquí se presenta). Finalmente, luego de acoger las sugerencias y observaciones del juicio de personal experto, se obtuvo la encuesta final que constó de cinco cuestiones dirigidas a explorar las opiniones de los 17 sujetos expertos respecto de nuestra problemática:

- Cuestión 1. ¿Cuáles son los aspectos positivos de los actuales programas de formación de profesorado de matemáticas de enseñanza básica y media en Chile?

- Cuestión 2. ¿Cuáles son los aspectos negativos o principales dificultades que actualmente atraviesan los programas de formación de profesorado de matemáticas de enseñanza básica y media en Chile?

- Cuestión 3. ¿Cuáles son los aspectos que son indispensables, y que deberían de ser considerados en los programas de formación inicial de profesorado de matemáticas de enseñanza básica y media?

- Cuestión 4. ¿Evalúa la Prueba Inicia los aspectos que usted señaló en su respuesta anterior? Y en general, ¿evalúa la Prueba Inicia, conocimientos y competencias didáctico-matemáticas necesarias para el correcto desempeño del futuro profesorado en su práctica docente?

- Cuestión 5. ¿De qué forma evaluaría si los egresados y egresadas de los programas de formación de profesorado poseen los conocimientos y competencias didácticas y matemáticas necesarias para su correcto desempeño en su práctica docente?

Es preciso señalar que, respetando la experticia de cada sujeto investigador y para garantizar la calidad de sus respuestas, como parte de la encuesta se les pidió que indicaran el nivel educativo para el cual pretendían responder las cuestiones (formación de profesorado de enseñanza básica, formación de profesorado de enseñanza media o para ambos niveles educativos). Asimismo, con la finalidad de profundizar en las reflexiones plasmadas en la encuesta, se les preguntó a los sujetos participantes si podían ser contactados para una entrevista personal o telefónica posterior, respondiendo afirmativamente la totalidad de participantes.

Para el análisis de las respuestas de participantes, utilizamos el método de análisis de contenido categórico propuesto por Leiblich, Tuval-Mashiach y Zilber (1998), donde las categorías representan los diversos temas o perspectivas que proporcionan un medio para la clasificación de palabras, frases o grupos de frases en una transcripción o respuesta escrita, en nuestro caso. Como señala Stemler (2001), dicho análisis de contenido es útil para examinar tendencias y patrones en los documentos escritos, lo que ayuda a proveer una base empírica para el seguimiento de las variaciones y posicionamientos en las opiniones públicas.

De esta forma, nuestras categorías fueron emergentes puesto que se establecieron después de examinar las respuestas escritas de los 17 sujetos participantes. En la siguiente sección presentamos los resultados del análisis del contenido categórico. 
UNICIENCIA Vol. 32, No. 1, pp. 68-88. Enero-Junio, 2018.

ISSN Electrónico: 2215-3470

URL: www.revistas.una.ac.cr/uniciencia

DOI: http://dx.doi.org/10.15359/ru.32-1.5

Email: revistauniciencia@una.cr

\section{Resultados}

Como se ha mencionado anteriormente, la encuesta se aplicó a 17 personas investigadoras chilenas expertas en la formación de profesorado de matemáticas, de las cuáles nueve han dirigido proyectos de investigación con financiamiento de alguno de los Programas de la Comisión Nacional de Investigación Científica y Tecnológica (CONICYT) de Chile. Las otras ocho han participado como colaboradoras en distintos proyectos sobre formación de profesorado llevados a cabo en Chile, adjudicados con fondos concursables.

Un aspecto importante de resaltar es que cada participante del estudio respondió a las preguntas sobre la base de los programas de formación de profesorado con los que estaban familiarizados (e.g., los programas de sus universidades). Este hecho nos permitió tener una mirada más amplia de lo que sucede con la formación inicial de profesorado a nivel nacional.

Por otro lado, a la pregunta sobre el nivel educativo para el cual responderían la encuesta, tres investigadores respondieron que solo para educación básica, cuatro respondieron solo para educación media y los diez restantes señalaron que responderían para ambos niveles educativos. A pesar de esto, al momento de analizar las respuestas con el fin de establecer las categorías, fue posible apreciar que los aspectos señalados por quienes respondieron solo para formación de profesorado de enseñanza básica, no eran distintos a los aspectos señalados por quienes respondieron para formación de profesorado de educación media y ambos niveles. Así, no fue necesario separar las categorías por niveles educativos, por lo que las categorías que se presentan a continuación, agrupadas de acuerdo con cada una de las cinco cuestiones planteadas, se establecieron sobre la base de las respuestas de la totalidad de investigadores e investigadoras participantes.

Cuestión 1: Aspectos positivos de los actuales programas de formación de profesorado

La primera pregunta planteada a los investigadores fue: ¿Cuáles son los aspectos positivos de los actuales programas de formación de profesorado de matemáticas de enseñanza básica y media en Chile? Reconocen ocho fortalezas en los actuales programas de formación inicial de docentes, que van desde aspectos curriculares hasta aspectos políticos y administrativos.

- Aumento de cursos disciplinares (de matemáticas). Según los sujetos participantes, uno de los puntos positivos de los actuales programas de formación es el aumento de los cursos de matemáticas, puesto que con dichos cursos se tiene la oportunidad de profundizar la comprensión de las matemáticas escolares que necesita conocer el futuro profesorado para posteriormente ser enseñadas. Uno de los investigadores lo plantea de la siguiente forma: “...pienso que han ido avanzando [los programas de formación] hacia una comprensión más profunda de las matemáticas que debe conocer el futuro profesor y que posteriormente tendrán que enseñar".

En sus respuestas, claramente refieren a lo que Shulman (1986; 1987), Hill, Ball y Schilling (2008) y el estudio TEDS-M (Tatto et al., 2008) denominan conocimiento del contenido. Sin embargo, como veremos más adelante, hay aspectos que no se están considerando en los cursos de matemáticas, que impiden que dichos conocimientos se logren con "profundidad y amplitud" (Schoenfeld \& Kilpatrick, 2008), lo cual se refleja, por ejemplo, en los resultados de la Prueba Inicia. Es decir, tal como señalan Pino-Fan y Godino (2015), es claro que la dimensión matemática del conocimiento didáctico-matemático del 
profesorado, que lo posibilita para resolver problemas y tareas matemáticas, no es suficiente para la práctica de enseñanza. Los diversos modelos del conocimiento del profesorado de matemáticas "coinciden en que además del contenido matemático, el profesor debe tener conocimientos sobre los diversos factores que influyen cuando se planifica e implementa la enseñanza de dicho contenido matemático" (Pino-Fan y Godino, 2015, p. 98).

- Incorporación de didácticas especificas. La incorporación de las denominadas didácticas específicas, por ejemplo, didáctica del álgebra, didáctica de la geometría, etc., representa, desde el punto de vista de los sujetos investigadores, una primera aproximación a la vinculación entre los cursos pedagógicos (generales) y los cursos disciplinares (de matemáticas). La opinión del siguiente investigador ejemplifica claramente el parecer de los participantes al respecto: “...uno de los principales aspectos positivos es la incorporación de las didácticas específicas, puesto que a través de ellas se hace un intento de integración de los cursos de pedagogía general con los de matemáticas, mediante la inclusión de la didáctica de las matemáticas en los programas".

- Intentos de articulación de la teoría con la práctica mediante experiencias de aula. Además de la inclusión de la didáctica de las matemáticas en los programas de formación, como un primer intento de vincular los conocimientos pedagógicos con los conocimientos matemáticos, los sujetos investigadores ven en la incorporación de las 'prácticas progresivas', un segundo intento de vinculación, pero ahora entre la teoría de la didáctica de la matemática con la práctica. Dichas prácticas progresivas, en algunos programas de formación se dan en tres momentos: prácticas tempranas, prácticas intermedias y prácticas finales (o profesionales). Como ejemplo de su opinión al respecto, dos investigadores señalan: “...un punto positivo es el intento de articular la formación didáctica (en matemáticas) con las experiencias reales de aula a partir de las prácticas de los estudiantes”, “...las prácticas tempranas en los programas se convierten en un gran escenario para que los futuros profesores observen y valoren el verdadero quehacer docente y así, desarrollar en él competencias". No obstante, aunque los sujetos investigadores valoran la inclusión de las horas de prácticas en los actuales programas de formación de profesorado, existe un consenso entre sí sobre el hecho de que la cantidad de horas designadas para las prácticas es insuficiente para el desarrollo de competencias necesarias para la profesión docente.

- Programas de formación basados en competencias. Uno de los investigadores lo manifiesta del siguiente modo: "La gran mayoría de los programas están basados en un enfoque por competencias, aunque estas se declaran sólo en los objetivos finales (o perfil de egreso) de los programas quedando, así, como una mera intencionalidad, pero esto ya es un primer paso”. Al respecto otro investigador plantea: “...aunque los programas señalan estar diseñados con base en competencias, lo cual es ya un avance, cabe preguntarse ¿qué tipo de competencias se espera desarrollar, competencias pedagógicas, competencias disciplinares (que permiten la resolución de problemas matemáticos) o competencias didáctico-matemáticas? En mi opinión los programas se han enfocado en los dos primeros grupos y no se han interesado por el desarrollo del tercer grupo de competencias que son las más importantes para nuestra actividad”. 
UNICIENCIA Vol. 32, No. 1, pp. 68-88. Enero-Junio, 2018.

URL: www.revistas.una.ac.cr/uniciencia

ISSN Electrónico: 2215-3470

Email: revistauniciencia@una.cr

- Programas acreditados. Con una duración promedio de 4 años, lo que los sujetos investigadores consideran un período adecuado para la capacitación del futuro profesorado. Asimismo, señalan las ventajas -bien conocidas en Chile- de tener programas acreditados.

- Aumento de personal académico con formación en educación matemática. De acuerdo con las personas encuestadas, la reciente incorporación de personal académico con formación postgradual en didáctica de las matemáticas -educación matemática, matemática educativa, etc.-, a los diversos programas de formación inicial de profesorado de las universidades, brinda un panorama alentador ante la situación actual. Uno de los participantes señala: “...este aumento de académicos con formación postgradual en el área, beneficia a esta mirada integradora de la didáctica de la matemática que se necesita en los programas de formación inicial”. Otro investigador anota: “...este punto positivo [la llegada de académicos con formación postgradual en el área], es el reflejo de la apuesta que años atrás realizó el Ministerio de Educación con la implementación de programas de becas para estudios postgraduales, cosa que debería mantenerse e inclusive aumentarse, pues esto permitiría que a mediano plazo consolidemos programas de formación inicial más integrados y apegados a la realidad del aula, con lo que tendríamos profesores mucho mejor preparados y competentes".

- Toma de conciencia de la necesidad de actualizar el conocimiento de los futuros profesores. Según los sujetos investigadores, hay un gran camino por recorrer en materia de la formación inicial del profesorado de matemáticas, pero que las instituciones encargadas de dicha formación reconozcan la necesitad de reorientar la formación del futuro profesorado, centrándola en el desarrollo de conocimientos y competencias didácticomatemáticas $-\mathrm{y}$ no solo en aspectos pedagógicos y matemáticos desvinculados-, es un primer gran paso.

\section{Cuestión 2: Principales dificultades de los actuales programas de formación de profesorado}

La segunda cuestión planteada a los participantes fue: ¿Cuáles son los aspectos negativos o principales dificultades que actualmente atraviesan los programas de formación de profesorado de matemáticas de enseñanza básica y media en Chile? Con base en las respuestas de los sujetos investigadores fue posible identificar ocho puntos débiles -incluso algunos investigadores señalaron que críticos- de los actuales programas de formación inicial de profesorado de matemáticas.

- Programas recargados de cursos. Señalaron que es necesario que los programas se autoevalúen con la finalidad de determinar cuáles son los cursos innecesarios para el desarrollo de competencias necesarias para la práctica docente. Además, señalaron que los cursos deberían estar articulados y con cada uno desarrollar competencias concretas y necesarias. Uno de los expertos plantea: “...uno de los puntos críticos es que los programas están recargados de cursos, hay muchos cursos de pedagogía, muchos cursos de matemáticas, totalmente desarticulados, muchos de ellos innecesarios para el contexto en el que se desempeñarán los futuros profesores". 
- Cursos con énfasis en lo teórico en detrimento de la práctica del profesorado. Sumado al problema señalado en el punto anterior, ponen énfasis en que la mayoría de los cursos 'innecesarios' son de características netamente teóricas, que poco o nada tienen que ver con la realidad del contexto en el que se desenvolverán los estudiantes de pedagogía. Uno de los expertos lo señala del siguiente modo: “....además de que esos cursos innecesarios tienen todo el énfasis en lo teórico, tanto en lo matemático como en educación”. Esta desarticulación entre el contenido de los cursos y el contexto o realidad escolar, es uno de los principales puntos críticos destacados por los sujetos participantes.

- Desarticulación entre las vertientes contempladas en los programas, y estas a su vez con la realidad del aula. Entre este grupo experto existe un acuerdo generalizado sobre el hecho de que, a pesar de los intentos de articulación de lo pedagógico con lo disciplinar, un problema que enfrentan los actuales programas de formación inicial de profesorado de matemáticas es la desarticulación entre las tres vertientes contempladas en dichos programas -cursos de pedagogía, cursos de matemáticas y cursos de didáctica de las matemáticas (didácticas específicas). Una de las personas expertas observa: “...tal vez pensemos que las prácticas profesionales son las encargadas de tal articulación, pero al parecer la realidad nos está demostrando que ellas [las prácticas profesionales] no alcanzan a lograrlo... entonces eso es un problema”. El equipo experto plantea, además, que los cursos de didácticas específicas se abordan desde un punto de vista "idealizado", y que estos se deben apegar más a la realidad y el contexto chileno.

- Poco espacio para analizar aspectos de la enseñanza de la matemática escolar. Otro aspecto en el que concuerdan es en la falta de espacios para reflexionar y profundizar en la matemática escolar, es decir, espacios para la comprensión de la matemática que realmente enseñará el profesorado. Al respecto, dos personas expertas comentan: "Los cursos contemplados en los programas de formación inicial de media y básica, no profundizan en los aspectos que sí son esenciales para la enseñanza y el aprendizaje de las nociones matemáticas, cómo utilizar la historia, cómo utilizar la tecnología, cómo plantear buenos problemas, qué metáforas son mejores para no inducir a conflictos cognitivos, etc. En media por ejemplo se ven aspectos matemáticos muy formales y se deja a un lado la idea que las nociones matemáticas siempre surgen con construcciones más empíricas y van avanzando a lo formal a lo largo del tiempo, estas miradas no se tienen en cuenta en el aula"; “...es importante una reflexión sobre la episteme que subyace a una idea matemática y cómo aporta la génesis histórica elementos sobre los procesos de construcción del saber matemático".

- Falta de espacios de reflexión sobre las prácticas (donde falta contextualización y vínculo con la realidad del aula). Otro aspecto bastante resaltado, y que está en relación con la problemática de la "saturación de cursos" es que en lugar de dichos cursos que consideran "innecesarios", los programas deberían contemplar espacios en los cuales el futuro profesorado tenga la oportunidad de analizar y reflexionar sobre la base de clases reales de matemáticas. Según los sujetos participantes, este hecho contribuiría al desarrollo de competencias didácticomatemáticas. En la sección 3.3 se profundizará esta idea, ya que también fue señalada por los sujetos investigadores como un elemento indispensable y que se debería considerar para el diseño de programas de formación de profesorado de matemáticas. 
- Estudiantes que ingresan a carreras de pedagogía con preparación insuficiente en matemáticas. La mayoría de las personas expertas señaló como un problema relevante el hecho de que estudiantes que ingresan a las carreras de pedagogía media en matemáticas o básica poseen una preparación insuficiente en matemáticas, lo que se evidencia con los bajos puntajes en la Prueba de Selección Universitaria (PSU).

- Falta de comprensión sobre la implementación del currículo por competencias. Aunque los sujetos participantes reconocen como un aspecto positivo de los programas de formación, el que estén diseñados con enfoques por competencias, también señalan como aspecto negativo el hecho de que el enfoque por competencias no se ha logrado concretar en Chile. Uno de los expertos lo señala del siguiente modo: “...la dificultad central tiene que ver con la escasa comprensión de que un currículo por competencias significa un verdadero cambio de paradigma, no basta con declararlo en los programas de estudio, este cambio se debe evidenciar en las metodologías de clase, en las evaluaciones, y en la manera en que se conciben y planifican las prácticas progresivas”.

- Falta de personal académico (formador) con especialidad en didáctica de la matemática. Los sujetos expertos señalan también un problema latente que tiene que ver con el perfil de personal formador de docentes de matemáticas de Chile. Si bien es cierto que, recientemente, en las universidades ha habido un incremento de personal académico con postgrado en Didáctica de la Matemática (Educación Matemática, Matemática Educativa, o afín), aún hay mucho personal formador de profesorado con perfiles de profesionales de las matemáticas, ingenierías o carreras afines, quienes focalizan su enseñanza en la matemática teórica, sin considerar los aspectos relacionados con la enseñanza de la matemática escolar. Una persona participante señala: “...aunque la mirada de los matemáticos es importante, hay una etapa en la formación de los profesores en la que los elementos teóricos y formalistas no aportan mucho al desarrollo de competencias que verdaderamente necesita un profesor. Además, está demostrado en la investigación que el conocimiento profundo de las matemáticas no es suficiente para la actividad de enseñanza”.

\section{Cuestión 3: Aspectos a considerar en los programas de formación de profesorado}

La tercera cuestión trataba de indagar sobre los aspectos o características que en la actualidad no se contemplan; pero deberían ser considerados en los programas de formación de profesorado de matemáticas. Concretamente, la cuestión fue: ¿Cuáles son los aspectos que son indispensables, y que deberían de ser considerados en los programas de formación inicial de profesorado de matemáticas de enseñanza básica y media? Con base en las respuestas fue posible identificar seis aspectos a considerar:

- Dominio del conocimiento matemático a enseñar. Una respuesta que da cuenta del sentir de los sujetos participantes al respecto es la siguiente: “...es fundamental que los futuros profesores manejen con profundidad las matemáticas que van a enseñar, es decir, que su comprensión del contenido les permita comprender la diversidad de estrategias, algoritmos, procedimientos, argumentos, etc., que les permita transmitir a los alumnos una visión amplia de las matemáticas y no como un conjunto de reglas sin sentido...”. En las respuestas es posible identificar que el grupo experto refiere a lo que Ball, Thames 
ISSN Electrónico: 2215-3470

DOI: http://dx.doi.org/10.15359/ru.32-1.5
UNICIENCIA Vol. 32, No. 1, pp. 68-88. Enero-Junio, 2018.

URL: www.revistas.una.ac.cr/uniciencia Email: revistauniciencia@una.cr

y Phelps (2008) denominan 'conocimiento especializado del contenido' al cual refieren como el "conglomerado de conocimientos y habilidades matemáticas exclusivas para la enseñanza" (p.400). Hill, Ball y Schilling (2008) indican que dicho conocimiento incluye "cómo representar con exactitud ideas matemáticas, ofrecer explicaciones matemáticas de reglas y procedimientos que comúnmente se encuentran en la enseñanza, analizar y comprender los métodos inusuales que permiten resolver un problema” (p. 377).

A lo anterior Schoenfeld y Kilpatrick (2008) lo llaman 'conocer las matemáticas escolares con profundidad y amplitud', y puntualizan:

El profesor tiene múltiples maneras de conceptualizar el contenido del nivel correspondiente, representarlo de diversas maneras, comprender los aspectos clave de cada tópico, y ver conexiones con otros tópicos del mismo nivel. El conocimiento profundo del contenido le permite seleccionar las 'grandes ideas' para ser propuestas a los alumnos, así como responder con flexibilidad a las cuestiones que le planteen. (p. 322)

Por su parte, Pino-Fan y Godino (2015) añaden a lo anterior el componente históricoepistemológico de los objetos matemáticos, lo que denominan 'significado holístico de referencia'. Estos autores señalan:

El profesor, además de las matemáticas que le permiten resolver problemas, deben tener cierta dosis de conocimiento matemático 'perfilado' para la enseñanza; es decir, el profesor debe ser capaz de movilizar diversas representaciones de un objeto matemático, resolver la tarea mediante distintos procedimientos, vincular el objeto matemático con otros objetos matemáticos del nivel educativo en el que se enseña o de niveles anteriores y posteriores, comprender y movilizar la diversidad de significados parciales para un mismo objeto matemático (que integran el significado holístico de referencia para dicho objeto), proporcionar diversas justificaciones y argumentaciones, e identificar los conocimientos puestos en juego durante la resolución de una tarea matemática. (p. 99)

Así, de acuerdo con estos autores, una comprensión profunda de las matemáticas a enseñar, puede permitir al futuro profesorado responder preguntas del tipo: “¿Además de tu solución, existe otra forma de resolver la tarea? ¿Cómo explicarías la solución de la tarea a un estudiante que no ha podido resolverla por los procedimientos vistos en clase? ¿Qué conocimientos se ponen en juego al resolver la tarea?” (Pino-Fan y Godino, 2015).

- Articulación de la disciplina con la práctica del aula. Los sujetos investigadores también señalan que es importante articular de forma congruente las tres vertientes contempladas actualmente en los programas de formación: pedagogía, matemáticas y didáctica de las matemáticas. También enfatizan que es necesaria la inclusión de más horas de prácticas -bien dirigidas e integradas- que permitan: primero la articulación de las tres vertientes antes señaladas con la realidad del aula; y segundo, el desarrollo de competencias didáctico-matemáticas enfocadas a que el futuro profesorado pueda planificar $y$, posteriormente, gestionar los aprendizajes de sus estudiantes. Estas sugerencias del personal experto van encaminadas a la consideración de diversos componentes que han 
sido ampliamente estudiados y discutidos en el campo de investigación sobre formación de docentes. Un primer componente o categoría es la propuesta de Grossman (1990) sobre el 'conocimiento del contexto':

Los profesores deberían basarse en su comprensión del contexto particular en el que enseñan para adaptar su conocimiento general a las necesidades específicas de la escuela y de cada uno de los estudiantes. El conocimiento del contexto incluye: conocimiento de los distritos en que los profesores trabajan, incluyendo las oportunidades, expectativas y limitaciones planteadas por el distrito; conocimiento del entorno de la escuela, incluyendo la 'cultura' de la escuela, directrices departamentales, y otros factores contextuales en el nivel de la escuela que afectan la instrucción; y conocimiento de estudiantes y comunidades específicos, y los antecedentes de los estudiantes, puntos fuertes, debilidades e intereses. (p. 9)

Con el conocimiento del contexto el futuro profesorado tendrá un punto de partida para la correcta planificación y adecuación de sus clases.

Otros componentes a considerar en este punto son: el conocimiento 'pedagógico del contenido' (Grossman, 1990; Hill, Ball \& Schilling, 2008; Shulman, 1986); "conocer a los estudiantes como personas que piensan y aprenden, diseñar y gestionar entornos de aprendizaje, y construir relaciones que apoyen el aprendizaje" (Schoenfeld \& Kilpatrick, 2008, p. 338); elementos de 'transformación y conexión' (Rowland, Huckstep \& Thwaites, 2005; Turner \& Rowland, 2011); y en general, las facetas 'cognitiva, afectiva, interaccional, mediacional y ecológica' que propone el modelo del conocimiento didáctico-matemático del profesor (PinoFan \& Godino, 2015; Pino-Fan, Assis \& Castro, 2015).

- Espacios de reflexión sobre la práctica y su relación con la gestión de los aprendizajes. De acuerdo con los sujetos encuestados, un aspecto que hasta ahora no se tiene en cuenta en los programas de formación, tiene que ver con espacios dirigidos a que el futuro profesorado adquiera competencias de análisis de las clases (o análisis didácticos). El grupo experto señala que dichos espacios, bien planificados y organizados, contribuirían al desarrollo de competencias que permitan al profesorado analizar sus clases, detectar errores o conflictos y buscar mejoras potenciales de sus prácticas. Schoenfeld y Kilpatrick (2008) lo llaman 'reflexionar sobre la propia práctica’:

Lograr proficiencia en la enseñanza de las matemáticas, como lograr proficiencia matemática, es un proceso interactivo a lo largo de la vida. Ante un problema de la práctica de la enseñanza, el profesor de matemáticas necesita pensar reflexivamente sobre el problema si quiere resolverlo. Una vez hecha habitual, la reflexión puede llegar a ser el principal mecanismo para mejorar la propia práctica. (p. 348)

Por su parte, el modelo del conocimiento didáctico-matemático (CDM) propone la dimensión 'meta didáctico-matemática' (Pino-Fan \& Godino, 2015), la cual contempla herramientas teórico-metodológicas concretas para llevar a cabo el proceso de reflexión y mejora de la propia práctica. Estos autores señalan: 
Somos conscientes de que el profesor es un sujeto reflexivo, racional, que toma decisiones, emite juicios, tiene creencias y genera rutinas propias de su desarrollo profesional ( $\underline{\text { Schön, }}$ 1983), y que los pensamientos del profesor influyen sustancialmente en su conducta e incluso la determinan (Schön, 1987). En síntesis, que el profesor debe tener las bases necesarias para reflexionar sobre su propia práctica. Por esta razón, para cada una de las facetas contempladas en la dimensión didáctica del CDM, se han previsto criterios de idoneidad que permiten al profesor reflexionar sobre su propia práctica y determinar mejoras potenciales de la misma. El desarrollo de los criterios de idoneidad, puede encontrarse en los trabajos de Godino (2011) y Godino, Batanero, Rivas y Arteaga (2013). (p. 103)

- Declaración explícita de los objetivos y competencias a desarrollar en cada asignatura. Como vimos en las secciones referentes a los resultados de las cuestiones 1 y 2 , aunque las personas investigadoras señalan como aspecto positivo el hecho de que la mayoría de los programas de formación estén basados en competencias, también advierten una incongruencia en la puesta en marcha de los currículos por competencias (falta de comprensión sobre la implementación del currículo por competencias). Para remediar esta 'incongruencia' aparente, el grupo experto señala que cada curso contemplado en los programas debe estar orientado al desarrollo de competencias específicas, las cuales deben ser congruentes, articuladas y declaradas en los planes de estudio de cada asignatura. La respuesta del siguiente participante ejemplifica la opinión generalizada: “...cada programa debería declarar qué competencias se espera que desarrollen los estudiantes con cada asignatura, con una caracterización de éstas y sus respectivos niveles de desarrollo...."

- Estudio sobre los recursos materiales, tecnológicos, etc. que favorezcan la gestión de los aprendizajes en el aula. Aunque este aspecto podría considerarse como una subcategoría del segundo aspecto -articulación de la disciplina con la práctica del aula-, más de la mitad de los sujetos encuestados apuntó que el estudio del correcto uso y adecuación de los recursos materiales y tecnológicos, que considere las particularidades de las nociones matemáticas, es un tema que no debería trivializarse. Este aspecto tiene que ver con el tercero de cuatro componentes involucrados en el 'conocimiento del contenido pedagógico’ de Grossman (1990, p. 8): “El tercer componente incluye conocimiento de los materiales curriculares disponibles para la enseñanza de un contenido particular, así como el conocimiento sobre el currículum horizontal y vertical para un tema”. También el modelo propuesto por Pino-Fan y Godino (2015) contempla este aspecto:

...la faceta mediacional del modelo del conocimiento didáctico-matemático del profesor, refiere a los conocimientos que debería tener un profesor para usar y evaluar la pertinencia del uso de materiales y recursos tecnológicos para potenciar el aprendizaje de un objeto matemático específico, así como la asignación del tiempo a las distintas acciones y procesos de aprendizaje. (p. 101)

- Mayor puntaje en la PSU como condición de ingreso a los programas. Siete de los sujetos participantes señalaron que un aspecto que deberían considerar los programas de formación de profesorado de matemáticas es la exigencia de un mayor puntaje en la PSU para el ingreso, lo que según ellos garantizaría, por un lado, que las personas postulantes 
posean los conocimientos previos mínimos requeridos para comenzar su formación y, por otro, no se perdería tanto tiempo del programa en enseñar los conocimientos previos (disciplinares) que debieron adquirir durante la etapa escolar.

\section{Cuestión 4: Consideraciones sobre la Prueba Inicia}

La cuarta cuestión planteada fue: ¿Evalúa la Prueba Inicia los aspectos que usted señaló en su respuesta anterior? Y en general, ¿evalúa la Prueba Inicia conocimientos y competencias didácticomatemáticas necesarias para el correcto desempeño del futuro profesorado en su práctica docente? Las respuestas del grupo experto encuestado dejaron ver de manera contundente un consenso sobre dos aspectos:

- Evalúa sólo aspectos disciplinares (teóricos). Algunas respuestas que ejemplifican este punto son las siguientes: “...la prueba inicia está muy enfocada en evaluar conocimientos netamente disciplinares...”; “...la prueba inicia está confeccionada en función de los Estándares Profesionales, los cuales abordan aspectos principalmente disciplinarios."; “...evalúa si los egresados de las carreras de pedagogía poseen conocimientos teóricos de matemáticas y de pedagogía en general."; “... [la prueba inicia] revisa aspectos de la capacidad de resolver problemas de matemáticas, no evalúa si sabe enseñar matemáticas..."; "Definitivamente no evalúa conocimientos matemáticos-didácticos como los que señalé en la pregunta anterior, sólo evalúa si el profesor sabe matemáticas y pedagogía teórica”.

- Proporciona un diagnóstico de la realidad de la formación inicial de profesorado. El segundo aspecto en el que están de acuerdo los sujetos participantes refiere al hecho de que la Prueba Inicia evidencia que los grupos egresados, a pesar de la saturación de cursos pedagógicos y disciplinares -ver resultados de la cuestión dos-no dominan ni las matemáticas ni la pedagogía. En palabras de dos investigadores: “...actualmente la prueba inicia está danto un diagnóstico de la realidad de la formación inicial de profesores, dejando en evidencia las falencias de lo que se está haciendo en las instituciones de formación de profesores..."; “...una ventaja que tiene actualmente la prueba inicia, es que nos permite reflexionar sobre qué es realmente lo que se está haciendo en los programas de formación inicial de profesores de matemáticas, los egresados no comprenden nociones matemáticas clave..."

\section{Cuestión 5: Sobre la evaluación de la formación de los grupos recién egresados de los programas de formación inicial}

La quinta cuestión fue planteada con la finalidad de explorar sugerencias del personal experto, para evaluar conocimientos y competencias didáctico-matemáticas. La pregunta fue: ¿De qué forma evaluaría si los grupos egresados de los Programas de Formación de Profesorado poseen los conocimientos y competencias didácticas y matemáticas necesarias para su correcto desempeño en su práctica docente? Aunque la mayoría de personal experto señaló que el diseño de instrumentos para este fin es un tema de investigación abierto y que va de la mano con la actualización de los programas de formación, sugirió los aspectos en los que se deben centrar los instrumentos, y las estrategias para recolectar datos para la evaluación de dichos aspectos. 
ISSN Electrónico: 2215-3470

DOI: http://dx.doi.org/10.15359/ru.32-1.5
UNICIENCIA Vol. 32, No. 1, pp. 68-88. Enero-Junio, 2018. URL: www.revistas.una.ac.cr/uniciencia Email: revistauniciencia@una.cr

- Conocimiento didáctico-matemático. El primer aspecto sugerido fue que los instrumentos deben evaluar conocimientos didáctico-matemáticos, y no conocimientos pedagógicos y matemáticos por separado. Hacen referencia principalmente a los aspectos discutidos en las dos primeras viñetas de los resultados de la cuestión 3.

- Competencias para la gestión de los aprendizajes en el aula. También señalaron que es indispensable evaluar los conocimientos didáctico-matemáticos aplicados en situaciones de aula reales. Para hacer sugieren varias alternativas: "Los egresados indiscutiblemente deben ser evaluados en la práctica profesional"; "Considero que las instituciones formadoras deberían de ser responsables de evaluar el desempeño de los futuros profesores, poniendo énfasis en el período de prácticas profesionales y el primer año de inserción laboral”; “...la evaluación de competencias didáctico-matemáticas debe hacerse en la práctica, mediante un seguimiento al egresado".

- Competencias para realizar análisis didácticos. Refieren a la evaluación de competencias de reflexión sobre la práctica. Principalmente refieren a los aspectos discutidos en la tercera viñeta de la sección 3.3. Para evaluar este tipo de competencias sugieren: “...filmaría sus clases, y por medio de pautas especialmente diseñadas, observaría cómo los futuros profesores evalúan las debilidades y fortalezas, y adaptan una propuesta didáctica"; "Grabación de episodios de clase, los estudiantes tendrían que realizar análisis didáctico de dicha clase buscando mejoras. Se tendrían que crear pautas para este fin".

\section{Reflexiones finales}

En este artículo hemos presentado una panorámica de las perspectivas y reflexiones de 17 sujetos investigadores expertos en el área de la formación de profesorado, respecto de las debilidades y fortalezas de los actuales programas de formación inicial de docentes de matemáticas de Chile. Los resultados de la encuesta otorgan información relevante para la evaluación del proceso de implementación de políticas nacionales como los estándares orientadores y la Prueba Inicia y, a la vez, nos permiten aproximarnos a la respuesta de la pregunta: ¿Cuál es la situación actual y hacia dónde debemos avanzar en materia de la formación inicial de profesorado de matemáticas en Chile?

Respecto de: ¿cuál es la situación actual?, que se relaciona con la pregunta: ¿cuáles son los problemas que enfrentan los actuales programas de formación de profesorado y cómo pueden éstos ser superados?, podemos decir que la mayoría del grupo experto consultado señala que a pesar de lo positivo que ha sido para los programas de formación inicial la incorporación de la didáctica de las matemáticas (o didácticas específicas), detectan, al mismo tiempo, la desarticulación entre los cursos de matemáticas y aquellos dedicados a la enseñanza de la disciplina (o cursos pedagógicos). Además, los cursos específicos de didáctica de la matemática (o educación matemática) considerados son escasos, de modo que la articulación con las prácticas progresivas no se realiza. Desde su perspectiva, esto se debe a la falta de espacios para la reflexión posterior a las prácticas (reflexión sobre la propia práctica). Una parte sugiere la incorporación de 'talleres' focalizados al análisis didáctico, para que el futuro profesorado se apropie de algunos elementos teóricos de la didáctica matemática que le permita definir criterios y adquirir competencias propias del análisis didáctico. 
Con respecto a la desarticulación entre la teoría y la práctica, señalan que esta puede provenir del hecho de que aún no se ha visualizado la necesidad de involucrar al estudiantado en las prácticas desde muy al inicio de su formación, pues se valora poco la experiencia del estudiante versus la teoría y la experiencia de otras personas, por ejemplo, la experiencia de sus docentes (Russel, 2014).

En relación con el tema del ingreso con bajos puntajes en la PSU, el panorama podría resultar un poco más desolador, puesto que la actual carrera docente (ley promulgada recientemente en marzo 2016), si bien señala que las exigencias irán en aumento con el pasar del tiempo, inicialmente solo demanda a los postulantes encontrarse en el percentil 50 para ingresar a las carreras de pedagogías ${ }^{1}$.

Refieren también a la falta de comprensión del significado de un currículo por competencias, lo que incide en su implementación. Otro punto que destacan es el hecho de que no todo el personal académico a cargo de los cursos de formación de profesorado de matemáticas es especialista en didáctica de las matemáticas, y quienes no lo son, ponen énfasis en el estudio teórico de la disciplina.

Es claro que ha habido un cambio en la mirada sobre el papel del docente en la sociedad chilena, su formación inicial y perfeccionamiento posterior, pero las exigencias son mínimas. Estamos en una situación en la que se ha tomado conciencia institucional de la problemática subyacente a la formación de profesores a nivel nacional, y el tipo de propuestas y medidas que ha adoptado el Ministerio de Educación de Chile, son cruciales y abren nuevas posibilidades para la mejora de la formación, en matemáticas y su didáctica, de los futuros profesores. La comunidad chilena de investigación en educación matemática debe trabajar en dichas mejoras, avanzando aún más hacia los alcances de la implementación de los Estándares Orientadores para carreras de Pedagogía en Educación Básica y Media en Matemáticas en las aulas universitarias de las carreras de pedagogía.

En lo referente a, ¿hacia dónde debemos avanzar?, estamos seguros de que el país tiene grandes oportunidades de mejorar la formación inicial de los docentes que enseñan matemática en las escuelas. Existe consciencia de los principales aspectos que las líneas de investigación y desarrollo nacionales e internacionales sugieren como relevantes para la formación de los profesores (Shulman, 1986; 1987; Rowland et al., 2005; Ball et al., 2008; Hill et al., 2008; Schoenfeld \& Kilpatrick, 2008; Tatto et al., 2008; König et al., 2014; Kaiser et al., 2014; Pino-Fan et al., 2015; Morales-López, 2017). Por ejemplo, los expertos nacionales destacan la relevancia de que los profesores dominen de manera 'profunda' el contenido matemático que enseñarán, que desarrollen competencias didáctico-matemáticas o dominen aspectos relacionados con el conocimiento pedagógico del contenido. Asimismo, enfatizan que los programas de formación de profesores deben brindar oportunidades para que los futuros docentes apliquen estos conocimientos en las prácticas de aula y tengan espacio para reflexionar sobre lo que ahí ocurre, no solo desde una perspectiva pedagógica general, sino también desde los aspectos didácticomatemáticos que son relevantes para desarrollar una clase de matemática eficaz. Esto quiere decir que, en conjunto, los expertos en educación matemática de nuestro país reconocen las dos principales corrientes internacionales relacionadas con el conocimiento pedagógico del

1 Ver información en el sitio Web de Política Nacional Docente, sección Formación Inicial de Calidad. http:// www.politicanacionaldocente.cl 
contenido o didáctico-matemático: 1) la que entiende este tipo de conocimientos desde una perspectiva cognitiva, con una cantidad determinada de subcomponentes que son evaluables en una prueba de lápiz y papel; y 2) aquella que la investigación de este tipo de conocimientos señala que sólo tiene sentido desde una perspectiva situada en el contexto en el que el (futuro) profesor utiliza estos conocimientos y otros aspectos relacionados para tomar decisiones de la gestión de clases y que, por lo tanto, debe ser evaluada también de manera situada (Depaepe et

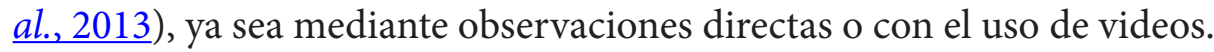

Por otro lado, las visiones de los investigadores indican que la Prueba Inicia requiere de cambios fundamentales para hacerse cargo de evaluar todos los componentes del conocimiento que requiere un profesor para ser competente en su labor, tomando en cuenta los resultados de múltiples investigaciones nacionales e internacionales que indican que no solo los conocimientos disciplinares y de pedagogía general son relevantes para la enseñanza, sino que un componente esencial es el conocimiento didáctico-matemático aplicado en el aula.

En general, las conceptualizaciones e ideas relevantes acerca de qué aspectos se deben incorporar en la formación de profesores de matemáticas, parecen estar adecuadamente instalados, al menos en una parte de los formadores de profesores de nuestro país. El desafío parece recaer en la búsqueda de las formas apropiadas de implementar dichos aspectos en los programas de formación inicial de profesores, y en las oportunidades y recursos para hacerlo. Aparece como especial desafío el lograr la articulación de los diversos tipos de conocimientos y competencias que los estudiantes de pedagogía van adquiriendo durante su formación y la capacidad para aplicarlos en la práctica pedagógica.

Finalmente debemos señalar que las sugerencias y recomendaciones derivadas de la reflexión de los 17 expertos encuestados, son orientadoras y abren espacios de discusión sobre los currículos actuales de los programas de formación inicial de profesores. En consecuencia, estamos seguros de que las ideas destacadas en las respuestas de los expertos permitirían sentar las bases para la discusión de un diseño de modelo teórico nacional, amparado en la teoría aportes de las investigaciones nacionales e internacionales sobre el tema- y en la experiencia del país, que sustente el diseño y mejora de los programas de formación de profesores de matemáticas en Chile.

\section{Agradecimientos}

Este trabajo ha sido desarrollado en el marco de los proyectos de investigación FONDECYT de iniciación 11150014 (financiado por el CONICYT de Chile), R02/15 y R04/15 (Universidad de Los Lagos).

\section{Referencias}

Ávalos, B. (2001). Chile: Mejoramiento de la formación inicial de profesores: Conjunción de políticas nacionales e iniciativas institucionales. En E. C. Braslavsky, I. Dussel y P. Scaliter (Eds.), Los formadores de jóvenes en América Latina: Desafíos, experiencias y propuestas (pp. 49-58). Ginebra: UNESCO-OEI.

Ball, D. L., Thames, M. H., \& Phelps, G. (2008). Content knowledge for teaching. What makes it special? Journal of Teacher Education, 59(5), 389-407. doi: https://doi. org/10.1177/0022487108324554 
UNICIENCIA Vol. 32, No. 1, pp. 68-88. Enero-Junio, 2018.

ISSN Electrónico: 2215-3470

URL: www.revistas.una.ac.cr/uniciencia

Email: revistauniciencia@una.cr

Cádiz, P., \& Cordella, F. (2015). Prueba Inicia: Menos de un tercio de los egresados logra más de un $75 \%$ de respuestas correctas. T13. Recuperado de http://t13.cl/139124

Cisternas, T. (2011). La investigación sobre formación docente en Chile. Territorios explorados e inexplorados. Revista Calidad en la Educación, 35, 131-164. doi: https://doi.org/10.4067/ $\underline{\text { S0718-45652011000200005 }}$

Cisternas, T. (2007). Consideraciones para el análisis de la investigación sobre formación docente en Chile. Revista Pensamiento Educativo, 41(2), 189-206. Recuperado de http:// pensamientoeducativo.uc.cl/index.php/pel/article/view/423/860

Cohen, L., Manion, L., \& Morrison, K. (2011). Research methods in education. London and New York: Routledge.

Contreras, S. (2016). Pensamiento pedagógico en la enseñanza de las ciencias. Análisis de las creencias curriculares y sus implicancias para la formación de profesores de enseñanza media. Formación Universitaria, 9(1), 15-24. doi: https://doi.org/10.4067/ $\underline{\text { S0718-50062016000100003 }}$

Contreras-Sanzana, G., \& Villalobos-Clavería, A. (2010). La formación de profesores en Chile: una mirada a la profesionalización docente. Educación y Educadores, 13(3), 397-417. doi: https://doi.org/10.5294/edu.2010.13.3.5

Depaepe, F., Verschaffel, L., \& Kelchtermans, G. (2013). Pedagogical content knowledge: A systematic review of the way in which the concept has pervaded mathematics educational research. Teaching and Teacher Education, 34, 12-25. doi: https://doi.org/10.1016/j. tate.2013.03.001

Godino, J. D. (2011). Indicadores de la idoneidad didáctica de procesos de enseñanza y aprendizaje de las matemáticas. XIII Conferencia Interamericana de Educación Matemática (CIAEM-IACME). Recife, Brasil.

Godino, J., Batanero, C., Rivas, H., \& Arteaga, P. (2013). Componentes e indicadores de idoneidad de programas de formación de profesores en didáctica de las matemáticas. Revemat: Revista Eletrônica de Educação Matemática, 8(1), 46-74. doi: http://dx.doi. org/10.5007/1981-1322.2013v8n1p46

Grossman, P. (1990). The making of a teacher: Teacher knowledge and teacher education. New York and London: Teachers College Press.

Hill, H. C., Ball, D. L., \& Schlling, S. G. (2008). Unpacking pedagogical content knowledge of students. Journal for Research in Mathematics Education, 39(4), 372-400. Recuperado de http://www.nctm.org/Publications/journal-for-research-in-mathematics-education/2008/ Vol39/Issue4/Unpacking-Pedagogical-Content-Knowledge -Conceptualizing-andMeasuring-Teachers -Topic-Specific-Knowledge-of-Students/

Kaiser, G., Bloemeke, S., Busse, A., Doehrmann, M., \& Koenig, J. (2014). Professional knowledge of (prospective) mathematics teachers - its structure and development. In P. Liljedahl, C. Nicol, S. Oesterle \& Dr. Allan (Eds.), Proceedings of the Joint Meeting of PME 38 and PMENA 36 (Vol. 1, pp. 35-50). Vancouver: PME.

König, J., Blömeke, S., Klein, P., Suhl, U., Busse, A. \& Kaiser, G. (2014). Is teachers' general pedagogical knowledge a premise for noticing and interpreting classroom situations? A video-based assessment approach. Teaching and Teacher Education, 38, 76-88. doi: https:// doi.org/10.1016/j.tate.2013.11.004 
ISSN Electrónico: 2215-3470

DOI: http://dx.doi.org/10.15359/ru.32-1.5
UNICIENCIA Vol. 32, No. 1, pp. 68-88. Enero-Junio, 2018. URL: www.revistas.una.ac.cr/uniciencia Email: revistauniciencia@una.cr

Latorre, M. (2006). Nuevas miradas, viejos problemas: Las relaciones entre la formación inicial y ejercicio profesional docente. Foro Educacional, 10, 41-63.

Leiblich, A., Tuval-Mashiach, R., \& Zilber, T. (1998). Narrative research: Reading, analysis, and interpretation. Thousand Oaks, CA: Sage Publications. doi: https://doi. org/10.4135/9781412985253

Ministerio de Educación de Chile (Mineduc). (2012). Estándares orientadores para carreras de Pedagogía en Educación Media. Recuperado de http://www.cpeip.cl/index2. php? id seccion $=4650$ \&id portal $=41$ \&id contenido $=24753$

Morales-López, Y. (2017). Costa Rica: The Preparation of Mathematics Teachers. En A. Ruiz (Ed.), Mathematics Teacher Preparation in Central America and the Caribbean (pp. 39-56). Springer International Publishing. Recuperado de http://link.springer.com/ chapter/10.1007/978-3-319-44177-1 3

Pino-Fan, L., Assis, A., \& Castro, W. F. (2015). Towards a methodology for the characterization of teachers' didactic-mathematical knowledge. Eurasia Journal of Mathematics, Science \& Technology Education, 11(6), 1429-1456. doi: https://doi.org/10.12973/eurasia.2015.1403a

Pino-Fan, L., \& Godino, J. D. (2015). Perspectiva ampliada del conocimiento didácticomatemático del profesor. PARADIGMA, 36(1), 87-109. Recuperado de http://revistas.upel. edu.ve/index.php/paradigma/article/view/2662

Prueba Inicia se aplicará por primera vez a egresados de Pedagogía en Enseñanza Media. (2012). Emol, Nacional. Recuperado de http://www.emol.com/noticias/ nacional/2012/10/01/562655/prueba-inicia-se-aplicara-por-primera-vez-a-egresados-depedagogia-en-ensenanza-media.html

Rowland, T., Huckstep, P., \& Thwaites, A. (2005). Elementary teachers' mathematics subject knowledge: The knowledge quartet and the case of Naomi. Journal of Mathematics Teacher Education, 8(3), 255-281. doi: https://doi.org/10.1007/s10857-005-0853-5

Russell, T. (2014). La práctica en la formación de profesores: Tensiones y posibilidades en la experiencia de aprender a enseñar. Revista Estudios Pedagógicos, 40(Especial), 223-238. doi: https://doi.org/10.4067/S0718-07052014000200013

Schoenfeld, A., \& Kilpatrick, J. (2008). Towards a theory of profiency in teaching mathematics. In D. Tirosh, \& T. L. Wood (Eds.), Tools and processes in mathematics teacher education (pp. 321-354). Rotterdam: Sense Publishers.

Schön, D. (1983). The reflective practitioner. New York: Basic Books.

Schön, D. (1987). Educating the reflective practitioner. Toward a new desing for teaching and learning in the professions. San Francisco: Jossey-Bass Publishers.

Shulman, L. S. (1986). Those who understand: Knowledge growth in teaching. Educational Researcher, 15(2), 4-14. doi: https://doi.org/10.3102/0013189X015002004

Shulman, L. S. (1987). Knowledge and teaching: Foundations of the new reform. Harvard Educational Review, 57(1), 1-22. doi: https://doi.org/10.17763/haer.57.1.j463w79r56455411

Stemler, S. (2001). An overview of content analysis. Practical Assessment, Research \& Evaluation, 7(17). Recuperado de http://PAREonline.net/getvn.asp? $v=7 \& n=17$ 
UNICIENCIA Vol. 32, No. 1, pp. 68-88. Enero-Junio, 2018.

Tatto, M. T., Schwille, J., Senk, S., Ingvarson, L., Peck, R., \& Rowley, G. (2008). Teacher Education and Development Study in Mathematics (TEDS-M): Policy, practice, and readiness to teach primary and secondary mathematics. Conceptual framework. East Lansing, MI: Teacher Education and Development International Study Center, College of Education, Michigan State University.

Tejedor, F. (2000). El diseño y los diseños en la evaluación de programas. Revista De InvestigacióN Educativa, 18(2), 319-339. Recuperado de http://revistas.um.es/rie/article/ view/121021/113711

Turner, F., \& Rowland, T. (2011). The knowledge quartet as an organising framework for developing and deepening teachers' mathematics knowledge. In T. Rowland, \& K. Ruthven (Eds.), Mathematical knowledge in teaching (pp. 195-212). London and New York: Springer. doi: https://doi.org/10.1007/978-90-481-9766-8 12

UNESCO-OREALC (2012). Antecedentes y criterios para la elaboración de políticas docentes en América Latina y el Caribe. París - Santiago de Chile: Autor.

\section{(2) $\odot \Theta \Theta$}

La formación inicial de profesores en Chile: 'voces' de la comunidad chilena de investigación en Educación Matemática (Luis R. Pino-Fan y otros) por Revista Uniciencia se encuentra bajo una Licencia CreativeCommons Atribución-NoComercial-SinDerivadas 3.0 Unported. 